\title{
The Application of Minimum Spanning Tree Algorithm in the Water Supply Network
}

\author{
Fengxia Cong ${ }^{1, a}$, Ying Zhao ${ }^{2, b}$ \\ ${ }^{1}$ College of Foreign Languages, Bohai University, Jinzhou, P.R. China \\ ${ }^{2}$ Computer Center, Liaoning University of Technology, Jinzhou, P.R. China \\ ajzcfx1972@tom.com, ${ }^{\mathrm{b}}$ Igjsjzy8@163.com
}

Keywords: water supply network; mathematical model; minimum spanning tree; Kruskal algorithm

\begin{abstract}
The system of urban water supply network is the important lifeline project of the city. With the continuous development of social economy, people are no longer satisfied with water supply requirements, but to put forward higher requirements for the safety, reliability and economy of the water supply. Based on actual demands to solve the economic problems of water supply network to ensure the lowest costs in the laying the pipelines. First, establishing a mathematical model of water supply network, so we can use the knowledge of graph theory to solve this problem; from the above that, the minimum spanning tree was needed to establish to ensure that costs are the lowest in the case of pipeline connectivity. Then using the Kruskal algorithm to generate minimum spanning tree; finally, an example was analyzed to verify its practicality, and the algorithm solved the problem of water supply network in laying pipelines successfully.
\end{abstract}

\section{Introduction}

Water is the source of life, and is closely related to human survival. Water supply network is a water distribution system created by people, which is a vital part of water supply system. Water supply system in the order usually consists of water intake structures, water treatment structures, water supply pumping stations, adjustment structures, drainage pipes and water supply pipe network. The water supply network mainly refers to the urban water supply pipe network system, which is an important material base to protect the city people's life and develop production and construction [1]. Urban water supply pipe network system can be regarded as an important lifeline of urban engineering. The traditional view is that the water supply network's mission is to provide sufficient amount of water, the residents have enough water to use. However, with the continuous development of social economy, people put forward higher requirements for the safety, reliability and economy of the water supply. Specifically, in the process of water supply, the quality of supplied water is healthy or not, such as water pipe corrosion or other factors lead to water quality problems; after an earthquake or major disaster, the water supply network is reliable or not, urban water supply network can be normal without the occurrence of secondary disasters; pipe network not only to have above two characteristics, economic issues is the focus. In the case of the entire water supply network connectivity, we must to ensure the lowest costs that aim to produce hedge-fund-like returns at lower cost. So in recent years, research on the economic aspect of water supply network is increasingly attracted people's attention. Water supply system is an important infrastructure of the city, and it also is an important part of urban lifeline project, which plays an irreplaceable role in protecting economic development, ensuring social production and meeting human life [2].

\section{The Establishment of the Mathematical Model for Water Supply Network}

In laying city network, the street interchanges must be considered because the pipelines must along the street to lay. In case of that water supply network can connect all users to make it with the lowest costs. To achieve this goal, you must consider how to select and handle these interchanges. This problem is a serious problem. The solution of this problem can provide a standard for the 
laying of water supply network to ensure best design effect.

According to graph theory, the water supply network can be viewed as a graph. So the contents of the water supply network need to be translated into the language of graph theory to help solve the problem of water supply network costs. The language from pipelines to graph is described as follows [3]:

a) The water supply center and users in the planning area are referred to as nodes, the intersection of the street known as the intersections. The nodes and intersections are regarded as the vertices of graph. So the issue can be converted to the shortest path between each vertex, and each vertex must be connected indirectly or not indirectly.

b) The routes that may be laying between nodes and intersections can be considered as edges of the graph.

c) The sum of construction costs and operating costs of each line is regarded as the weights of edges. The sum of weights is the minimum that is the lowest costs, that is the purpose of the design you want to achieve.

Through the above three steps, the water supply network can form a graph, this graph includes the vertices, edges and weights. Using $G(V, E, W)$ to represent, $\mathrm{V}$ represents the set of vertices in the graph; $E$ represents the set of edges in the graph; W represents the set of weights of each edge in the graph.

Setting $\mathrm{T}$ is a spanning tree of diagram of $G$, then:

$\mathrm{W}(\mathrm{T})=\sum_{\mathrm{e}_{\mathrm{uv}} \in \mathrm{T}} \mathrm{W}_{\mathrm{uv}}$

Among them, $W(T)$ is the sum of weights in the tree of T; $e_{u v}$ is the any edge in the tree of T; $W_{u v}$ is the weights of $e_{u v}$. The purpose of design is to require the minimum values of $W(T)$. Only this way can ensure the lowest costs of laying pipelines.

In summary, the problem of the minimum costs of the water supply network may be as a problem of seeking minimum spanning tree in the graph. The minimum spanning tree must exist. According to the actual situation, each node will certainly connected when laying water supply network, so there will be a minimum spanning tree certainly. There are a variety of algorithms to generate minimum spanning tree, such as Prim algorithm, Kruskal algorithm and simple algorithm and so on [4].

\section{The Basic Concept of Algorithm}

Kruskal algorithm chooses the right edge according to the ascending order of weights to construct a minimum spanning tree. Kruskal algorithm, also known as avoidance circle method, starting from the shortest side, the edge attached to the tree does not form a loop, then the edge can be added to the tree, otherwise examine the next edge [5]. Specific steps are as follows:

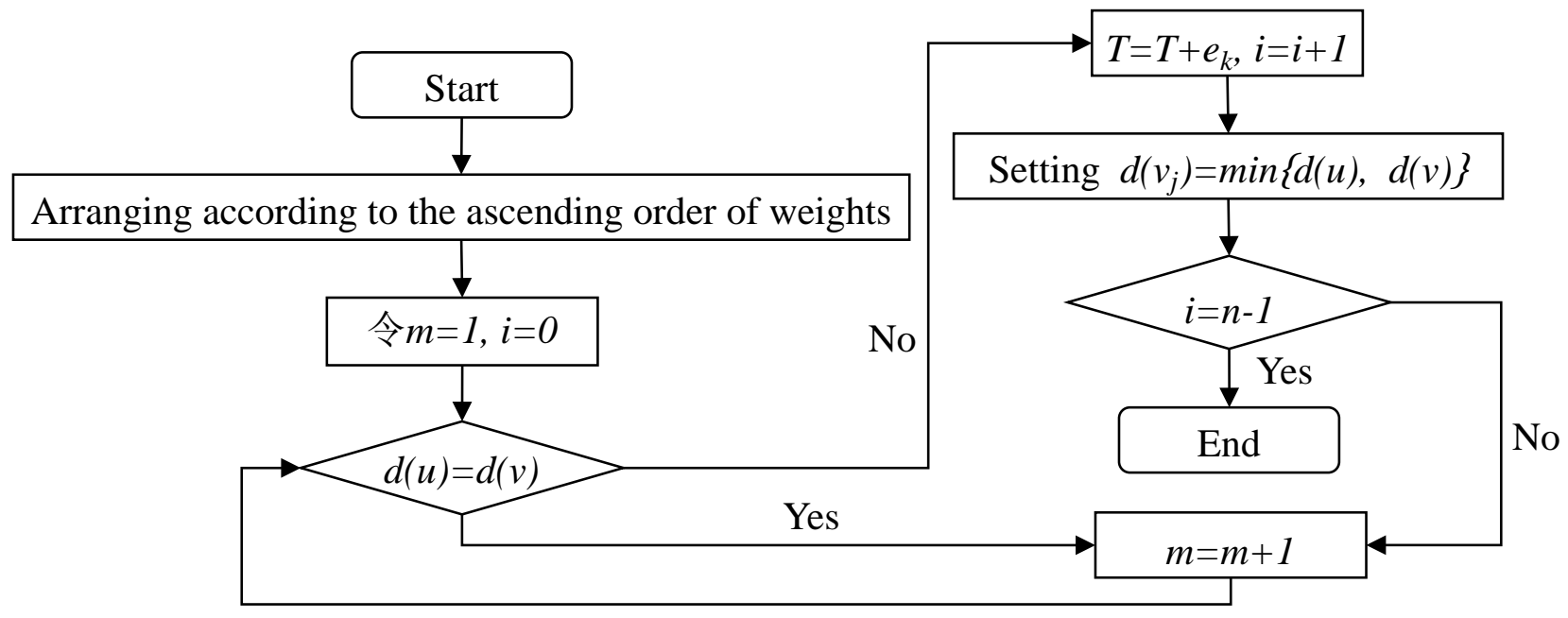

Fig. 1. The flow chart of the Kruskal algorithm

(1) Firstly, all vertices in connectivity network need to be added to minimum spanning tree to 
construct a forest; that $\mathrm{V}$ is the complete set, $E$ is the empty set;

(2) The edge set $E$ need to range according to the size of the weights, which requires $W\left(e_{1}\right) \leq W\left(e_{2}\right) \leq \cdots \leq W\left(e_{n}\right)$. Setting $T=V(G) ; d\left(v_{j}\right)=j, j=1,2, \cdots, n ; m=1, i=0$;

(3) Setting $e(m)=(u, v)$, if $d(u)=d(v)\left(d(u)=d(v) \leftrightarrow T+e_{k}\right)$, that says there is a circle, please turns to step (6); otherwise, setting $T=T+e_{k}, i=i+1$;

(4) For all the $v_{j}$ of meeting $d\left(v_{j}\right)=\max \{d(u), d(v)\}$, setting $d\left(v_{j}\right)=\min \{d(u), d(v)\}$;

(5) If $i=n-1$, the $T$ is minimum spanning tree, then program is end;

(6) Setting $m=m+1$, please turns to step (3).

The basic flow chart of the algorithm shows in Fig. 1.

\section{The Example Analysis}

A city wants to lay the pipelines of water supply network, the lines show in Fig. 2. As shown in Fig. 2, $v_{7}$ is the source of water supply. According to the above described steps, the weights of each edge need to range in ascending order, that is $W\left(e_{0}\right) \leq W\left(e_{1}\right) \leq \cdots \leq W\left(e_{10}\right)$.

First adding all the vertices and edge $e_{0}$ of the minimum weights, that shows in Fig. 3, and then the $e_{1}$ can be added to the figure to see whether the loop is formed, if loop is not formed, then add edge succeed; if the loop is formed, you need to withdraw edge $e_{1}$ to continue to add $e_{2}$, that shows in Fig. 4. And so on until the edge of $e_{10}$, the minimum spanning tree will be produced. That shows in Fig. 5.

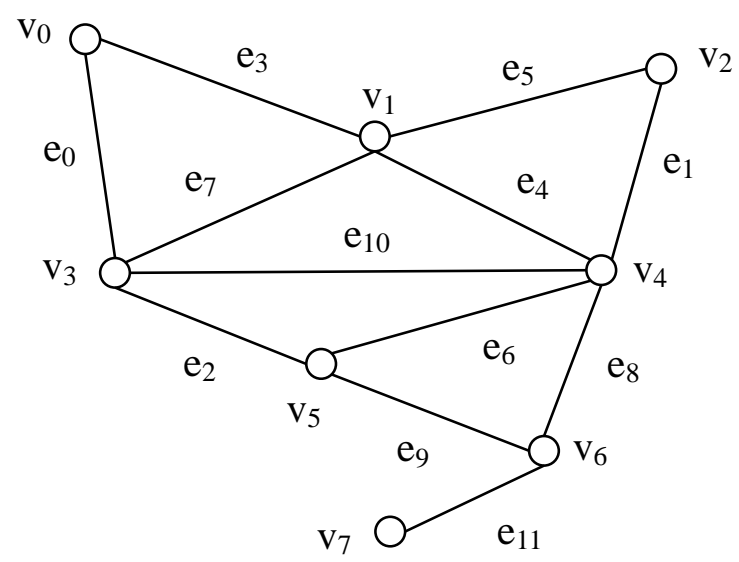

Fig. 2. Connectivity graph of water supply network

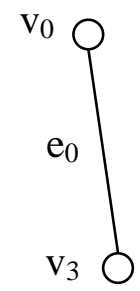

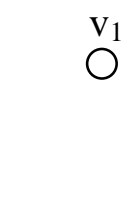

$\mathrm{V}_{5}$

$$
\mathrm{V}_{7}
$$

Fig. 4. The graph when first time cycling of algorithm

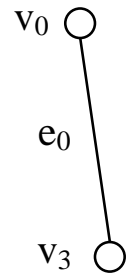

$\mathrm{V}_{1}$

$\mathrm{O}_{2}$

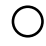

$\mathrm{O} \mathrm{v}_{4}$

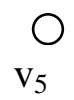

5

$$
\bigcirc v_{6}
$$

$\mathrm{V}_{7} \mathrm{O}$

Fig. 3. Weighted graph when initializing algorithm

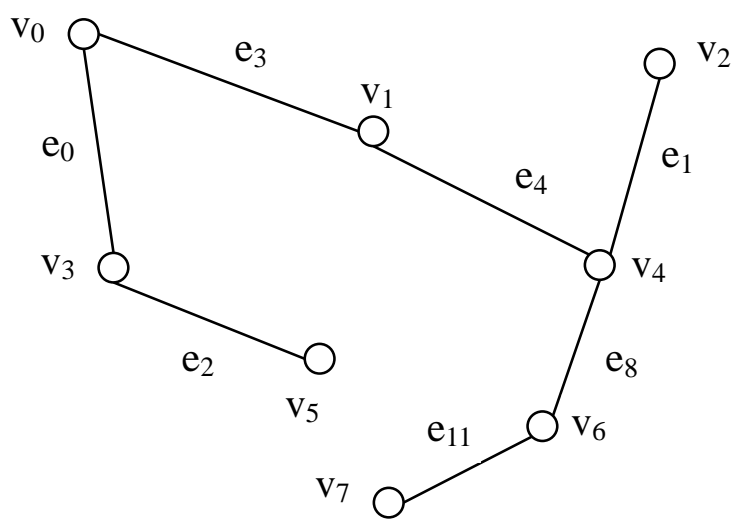

Fig. 5. Minimum spanning tree of city

The time complexity of Kruskal algorithm is mainly determined by the sorting method, the sorting algorithm is only concerned with the number of the edges, regardless of the number of vertices in the graph. In the process of solving the minimum spanning tree, its time complexity is analyzed [6]. When using sorting algorithm that the time complexity is $O(e \log e)$, the time 
complexity of Kruskal algorithm is also the $O(e \log e)$. Therefore, when the number of vertices of weighted graph is more and the number of edges is less, using Kruskal algorithm to generate minimum spanning tree that works best.

\section{Conclusion}

The city is the staging area of politics, culture, economy and others, and the water supply network is the lifeline of a city undoubtedly. Urban water supply network is the critical infrastructure to ensure a city's economic development and living standards, and it also is the city's lifeline for survival and development [7]. The problem of pipelines selection of water supply network has been one focus of the study. With economic development, the idea of the shortest path algorithm in graph theory was introduced to water supply network in this article in order to further meet the modern requirements of the water supply network. Minimum spanning tree can be formed by finding the shortest path [8]. The algorithm regards the lowest costs as objective function to generate minimum spanning tree. The example analysis verifies the practicality and economy of minimum spanning tree algorithm and the algorithm has some guidance for evaluation budget of project investment for water supply system. Minimum spanning tree not only applies to the water supply network, it is also used in many other places. For example: laying cables and wires, laying pipes and so on. The optimal routing paths can be calculated by using this algorithm to save time, save material and save labor.

\section{Acknowledgement}

This work is supported by 2014 general subject of colleges and universities scientific research of Liaoning province (L2014248): Emergency Disposal on Urban Water Supply Pipe Network Accident Based on FCR Computer Reasoning.

\section{References}

[1] P. Li, "Research on Water Quality Optimization Technique of Water Supply Network Based on Water Node Age," Master's Degree of Qingdao Technological University, 2013.

[2] X. F. Qing, "Prediction of the Economic Residual Life of Water Pipes," Master's Degree of Tianjin University, 2011.

[3] H. Mao, L. W. Xie, Z. X. Zhang, "Applying Pruning-based Minimum Spanning Tree Algorithm Water Supply Networks," Computer Applications and Software, vol. 28, no. 2, pp. 109-110, 2011.

[4] C. Y. Xue, "The Application of Minimum Cost Spanning Tree to Solve the Question of Urban Highway," Computer Programming Skills \& Maintenance, vol. 16, no. 6, pp. 30-31, 2009.

[5] Y. Chen, Q. Y. Han, "Applying an Improved Minimum-cost Spanning Tree Algorithm to Optimal Planning of Distribution Network Frame," Guangdong Electric Power, vol. 20, no. 3, pp. 6-11, 2007.

[6] W. Wang, S. Y. Meng, "Research and Improvement of Kruskal Algorithm," Journal of Chongqing University of Arts and Science (Natural Science Edition), vol. 29, no. 3, pp. 25-27, 2010.

[7] Y. Y. Lin, "Design of Real-time Monitoring System for Municipal Water Supply Network and Optimization of Monitoring Points," Master's Degree of South China University of Technology, 2013.

[8] L. Wang, "The Research of Application in Distribution Network Reconfiguration Based on Shortest Path Algorithm and Minimum Spanning Tree Algorithm," Master's Degree of Xi'an University of Technology, 2009. 